Research Article

XiuLin Huang*, Yan Xu, and ChengZhi Liu

\title{
An attempt to geometrize electromagnetism
}

https://doi.org/10.1515/phys-2018-0106

Received May 16, 2018; accepted Sep 06, 2018

\begin{abstract}
This study investigates the curved worldline of a charged particle accelerated by an electromagnetic field in flat spacetime. A new metric, which dependes on the charge-to-mass ratio and electromagnetic potential, is proposed to describe the curve characteristic of the worldline. The main result of this paper is that an equivalent equation of the Lorentz equation of motion is put forward based on a 4-dimensional Riemannian manifold defined by the metric. Using the Ricci rotation coefficients, the equivalent equation is self-consistently constructed. Additionally, the Lorentz equation of motion in the non-inertial reference frames is studied with the local Lorentz covariance of the equivalent equation. The model attempts to geometrize classical electromagnetism in the absence of the other interactions, and it is conducive to the establishment of the unified theory between electromagnetism and gravitation.
\end{abstract}

Keywords: Electromagnetism, Worldline, Riemannian Metric, Ricci rotation coefficients, Local Lorentz transformations

PACS: 03.50.De; 04.20.Cv; 02.40.Ky

\section{Introduction}

Since Einstein's general relativity succeeded in geometrizing gravity, numerous people have made great efforts to geometrize electromagnetism in lots of unified-field theories [1-8]. However, these unified-field theories are not widely accepted. In recent years, work on geometrization

\footnotetext{
${ }^{\star}$ Corresponding Author: XiuLin Huang: Changchun Observatory, National Astronomical Observatories, Chinese Academy of Sciences, Changchun, China; Guizhou Provincial Key Laboratory of Radio Astronomy and Data Processing; Email: huangxl@cho.ac.cn Yan Xu: Changchun Observatory, National Astronomical Observatories, Chinese Academy of Sciences, Changchun, China;

Email: xuy@cho.ac.cn

ChengZhi Liu: Changchun Observatory, National Astronomical Observatories, Chinese Academy of Sciences, Changchun, China; Email: lcz@cho.ac.cn
}

of electromagnetism without consideration of gravity has begun to increase [9-11]. Such studies are not complicated and the physical meaning is more straightforward. Moreover, the research on only geometrization of electromagnetism constantly contributes to establishing a final unified field theory.

Gravity is identified as a geometric phenomenon of a curved spacetime and the geometric phenomenon is the subject investigated in general relativity. Electromagnetism is essentially different from gravity, because it does not relate to the geometric phenomenon of the curved spacetime. Finding a suitable geometric phenomenon for electromagnetism is the first problem for geometrizing electromagnetism. In the previous references [12-15], the geometrical description of the worldlines of charged particles in homogeneous electromagnetic fields have been studied using Frenet-Serret formulae. The time-like worldline of a charged particle accelerated by an electromagnetic field is possibly a geometric phenomenon of geometrizing electromagnetism. When a particle is accelerated by an electromagnetic force in flat spacetime, the worldline of the particle becomes a curved line $x^{a}(\tau)$, and it is closely relate to the Lorentz equation of motion

$$
\frac{d^{2} x^{a}}{d \tau^{2}}=\frac{e}{m} F_{b}^{a} \frac{d x^{b}}{d \tau} .
$$

The geometrical characteristic of $x^{a}(\tau)$ may be the key to geometrize electromagneticsm. In this paper, we would like to provide an investigation on the geometrical characteristic of the worldline $x^{a}(\tau)$ from a different point of view.

The essential difference between the curved spacetime and the curved worldline indicates that there could be many curved worldlines at one spacetime point. To show the feature of the curved worldline, we structure "artificial" curvilinear coordinates $\left\{x^{\hat{a}}\right\}$ regarding the different metrics of the curved worldline $x^{a}(\tau)$. The coordinates $\left\{x^{\hat{a}}\right\}$ are not real curved spacetime, but are hypothetical curved coordinates associated with the flat coordinates $\left\{x^{a}\right\}$. Therefore, a metric could be obtained using the linear relationship between the flat differential coordinates $d x^{a}$ and the "artificial" differential coordinates $d x^{\hat{a}}$, which differs from the model that a metric is derived by the coordinates transformation [16-18]. In this current work, we attempt to introduce differential geometry by defining the 
new metric of the curved worldlines of a charge particle in an electromagnetic field, and then obtain an equivalent equation of the Lorentz equation of motion using differential geometry.

This paper is organized as follows. In Section 2, a new metric is given depending on the worldline of a charged particle in an electromagnetic field. In Section 3, an equivalent equation of the Lorentz equation of motion is proposed using the new metric. Section 4 discusses the local Lorentz covariance of the Lorentz equation of motion. Section 5 provides a conclusion.

\section{The new metric for classical electromagnetism}

The length element squared $d s^{2}$ of a worldline $x^{a}(\tau)$ in 4dimension flat spacetime is

$$
d s^{2}=\eta_{a b} d x^{a} d x^{b},
$$

where metric $\eta_{a b}$ is

$$
\eta_{a b}=\left(\begin{array}{cccc}
1 & 0 & 0 & 0 \\
0 & -1 & 0 & 0 \\
0 & 0 & -1 & 0 \\
0 & 0 & 0 & -1
\end{array}\right) .
$$

The differential coordinate $d x^{a}$ describes the motion of a charged particle in an electromagnetic field and 4-velocity of the charged particle is $U^{a}=\left(\frac{1}{\sqrt{1-v^{2}}}, \frac{v^{i}}{\sqrt{1-v^{2}}}\right)$, where $v^{i}=$ $\frac{d x^{i}}{d x^{0}}, i=1,2,3$ and the speed of light $c=1$. Because any curved worldline obeys the relation (2) in flat spacetime, the flat metric $\eta_{a b}$ does not reflect the curved properties of a worldline. In this model, using "artificial” curvilinear coordinates $x^{\hat{a}}$ we give a new metric for the worldline of the charged particle in an electromagnetic field and the effects of radiation are ignored.

Assuming that the transformation between differential coordinates $d x^{\hat{a}}$ and $d x^{a}$ is linear, the linear relationship at each point of the worldline is formulated as

$$
d x^{a}=h_{\hat{a}}^{a} d x^{\hat{a}} .
$$

Equations (2) and (4) show that the length element $d s^{2}$ is expressed with the differential coordinate $d x^{\hat{a}}$,

$$
d s^{2}=G_{\hat{a} \hat{b}} d x^{\hat{a}} d x^{\hat{b}},
$$

where

$$
G_{\hat{a} \hat{b}}=\eta_{a b} h_{\hat{a}}^{a} h_{\hat{b}}^{b} .
$$

The key issue of obtaining metric $G_{\hat{a} \hat{b}}$ is the relationship between differential coordinates $d x^{\hat{a}}$ and $d x^{a}$.
We consider that the linear transformation $h_{\hat{a}}^{a}$ needs to satisfy three limiting conditions. In the first limiting condition, in the absence of the case of electromagnetic interaction, the linear transformation $h^{a}{ }_{\hat{a}}$ should be $\delta_{\hat{a}}^{a}$. The second limiting condition is provided by the study of the Lorentz equation of motion (1), form which the worldline $x^{a}(\tau)$ depends on the charge-to-mass ratio $e / m$ and electromagnetic potential $A_{a}$. As a result, the linear transformation $h^{a}{ }_{\hat{a}}$ should be dependent on $e / m$ and $A_{a}$. The third limiting condition derives from the length element's magnitude change of the worldline, measured by the multiple static-observers positioned at each point of the worldline. The length element of the observers is

$$
d s^{\prime}=d x^{0},
$$

which is constant at each point of spacetime. comparing equations (2) and (7), one can find that:

$$
d s=\sqrt{1-v^{2}} d s^{\prime},
$$

which indicates that the length element's magnitude is proportional to $\sqrt{1-v^{2}}$. Thus, the linear transformation $h^{a}{ }_{\hat{a}}$ should show the characteristic of the length element's magnitude, which is the third limiting condition.

In our model, the linear transformation $h_{\hat{a}}^{a}$ is defined as:

$$
\begin{aligned}
h_{\hat{a}}^{0} & =\delta_{\hat{a}}^{0}\left(1-Q^{\prime}\right)+\frac{e}{m} B_{\hat{a}}, \\
h_{\hat{a}}^{i} & =\delta_{\hat{a}}^{i},
\end{aligned}
$$

where $Q^{\prime}=Q /\left(1-\frac{e}{m} A_{0}+Q\right)$ and $B_{\hat{a}}$ is expressed as

$$
B_{\hat{a}}=\frac{A_{a}\left(x^{a}\right)}{1-\frac{e}{m} A_{0}\left(x^{a}\right)+Q(t)},
$$

where $Q(t)$ is a function of time, $A_{a}\left(x^{a}\right)$ is electromagnetic potential and it is used to structure the equation (see Appendix)

$$
\frac{1}{\sqrt{1-v^{2}}}=1-\frac{e}{m} A_{0}+Q .
$$

Then, the inverse transformation $h_{a}{ }^{\hat{a}}$ of the transformation $h^{a}{ }_{\hat{a}}$ yields

$$
\begin{aligned}
h_{a}{ }^{\hat{0}} & =\delta_{a}^{\hat{0}}(1+Q)-\frac{e}{m} A_{a}, \\
h_{a}{ }^{\hat{i}} & =\delta_{a}^{\hat{i}} .
\end{aligned}
$$

The transformation $h_{\hat{a}}^{a}$ and the inverse transformation $h_{a}{ }^{\hat{a}}$ satisfy the orthogonal relationship

$$
h^{a}{ }_{\hat{a}} h_{b}{ }^{\hat{a}}=\delta_{b}^{a} \quad \text { and } \quad h_{\hat{a}}^{a} h_{a}{ }^{\hat{b}}=\delta_{\hat{a}}^{\hat{b}} .
$$

It is easily demonstrated that the transformation $h^{a} \hat{a}$ meets the first and second limiting conditions. Besides, using equation (11), we have

$$
h_{\hat{0}}^{0}=\sqrt{1-v^{2}}=1+\frac{e}{m} B_{\hat{0}}-Q^{\prime},
$$


which reflects the change of the length element's magnitude, thus the third limiting condition is satisfied. The new metric $G_{\hat{a} \hat{b}}$ of a worldline's length element is obtained by equation (6) and (9):

$G_{\hat{a} \hat{b}}=$

$$
\left(\begin{array}{cccc}
D_{\hat{0}} D_{\hat{0}} & \frac{e}{m} D_{\hat{0}} B_{\hat{1}} & \frac{e}{m} D_{\hat{0}} B_{\hat{2}} & \frac{e}{m} D_{\hat{0}} B_{\hat{3}} \\
\frac{e}{m} D_{\hat{0}} B_{\hat{1}} & \left(\frac{e}{m} B_{\hat{1}}\right)^{2}-1 & \left(\frac{e}{m}\right)^{2} B_{\hat{1}} B_{\hat{2}} & \left(\frac{e}{m}\right)^{2} B_{\hat{1}} B_{\hat{3}} \\
\frac{e}{m} D_{\hat{0}} B_{\hat{2}} & \left(\frac{e}{m}\right)^{2} B_{\hat{1}} B_{\hat{2}} & \left(\frac{e}{m} B_{\hat{2}}\right)^{2}-1 & \left(\frac{e}{m}\right)^{2} B_{\hat{2}} B_{\hat{3}} \\
\frac{e}{m} D_{\hat{0}} B_{\hat{3}} & \left(\frac{e}{m}\right)^{2} B_{\hat{1}} B_{\hat{3}} & \left(\frac{e}{m}\right)^{2} B_{\hat{2}} B_{\hat{3}} & \left(\frac{e}{m} B_{\hat{\jmath}}\right)^{2}-1
\end{array}\right),
$$

where $D_{\hat{0}}=h_{\hat{0}}^{0}=1+\frac{e}{m} B_{\hat{0}}-Q^{\prime}, B_{\hat{a}}=\frac{A_{a}}{1-\frac{e}{m} A_{0}+Q}, Q$ is a function of time and $A_{a}$ is the electromagnetic potential.

It can be seen that the length element $d s^{2}$ of the curved worldline $x^{a}(\tau)$ has two different expressions with different characteristics: $d s^{2}=\eta_{a b} d x^{a} d x^{b}$ and $d s^{2}=$ $G_{\hat{a} \hat{b}} d x^{\hat{a}} d x^{\hat{b}}$. In the first, $d s^{2}=\eta_{a b} d x^{a} d x^{b}$, flat metric $\eta_{a b}$ is invariant, which does not reflect the curvature of the worldline. The change of length element $d s^{2}$ at each point along the worldline depends on the differential coordinate $d x^{a}$, which can describe the motion of the particle. In the second expression, $d s^{2}=G_{\hat{a} \hat{b}} d x^{\hat{a}} d x^{\hat{b}}$, the "artificial" differential coordinate $d x^{\hat{a}}$ is regarded as invariant and it does not describe the motion of the particle. Besides, the change of length element $d s^{2}$ depends on the metric $G_{\hat{a} \hat{b}}$ at each point of the worldline.

\section{Differential geometry for electromagnetism}

In this section, we use the length element expression $d s^{2}=$ $G_{\hat{a} \hat{b}} d x^{\hat{a}} d x^{\hat{b}}$ to derive equations for the motion of a charged particle in an electromagnetic field based on differential geometry. Let $M$ be a 4-dimensional Riemannian manifold with metric $G_{\hat{a} \hat{b}}$, which is defined by equation (15) at the worldline of the charged particle and $G_{\hat{a} \hat{b}}(Q=0)$ at other space-time points. Now, the transformation $h^{a}{ }_{\hat{a}}$ is called tetrad or vierbein. We can derive the equation of a worldline from an action given in the following equation, which is proportional to the length of the worldline.

$$
S\left(x^{\hat{a}}\right)=\int \sqrt{G_{\hat{a} \hat{b}} \frac{d x^{\hat{a}}}{d \tau} \frac{d x^{\hat{b}}}{d \tau}} d \tau,
$$

where proper time $\tau$ is a parameter associated with a point on the worldline and the coordinate $x^{\hat{a}}$ is variable. Variation of the action (16) with respect to $x^{\hat{a}}$ provides the traditional geodesic equation

$$
\frac{d}{d \tau}\left(\frac{d x^{\hat{a}}}{d \tau}\right)+\Gamma_{\hat{b} \hat{c}}^{\hat{a}} \frac{d x^{\hat{b}}}{d \tau} \frac{d x^{\hat{c}}}{d \tau}=0,
$$

where $\Gamma^{\hat{a}}{ }_{\hat{b} \hat{c}}=\frac{1}{2} G^{\hat{a} \hat{d}}\left(\partial_{\hat{c}} G_{\hat{d} \hat{b}}+\partial_{\hat{b}} G_{\hat{c} \hat{d}}-\partial_{\hat{d}} G_{\hat{b} \hat{c}}\right)$ is Christoffel symbol on $M$. Equation (17) cannot describe the motion of the charged particle, because $d x^{\hat{a}} / d \tau$ is not the 4-velocity of the charged particle. We know that $d x^{a} / d \tau$ is considered to describe the motion of a charged particle, and the relationship between $d x^{a}$ and $d x^{\hat{a}}$ is given by linear transformation $h_{\hat{a}}^{a}$ in equation (4). Thus, according to the coordinates $x^{a}$, equation (17) is rewritten as [19]

$$
\frac{d^{2} x^{a}}{d \tau^{2}}+\omega^{a}{ }_{b c} \frac{d x^{b}}{d \tau} \frac{d x^{c}}{d \tau}=0,
$$

where $\omega^{a}{ }_{b c}$ represents the Ricci rotation coefficients of the tetrad $h_{\hat{a}}^{a}$. Besides, the tetrad $h_{\hat{a}}^{a}$ is also obtained using the metric $G_{\hat{a} \hat{b}}$ under "resting constraint conditions" [20]. In the torsion-free case, $\omega^{a}{ }_{b c}$ is given as reported in literature [21]

$$
\omega_{b c}^{a}=\frac{1}{2}\left(C_{b c}^{a}+C_{b c}{ }^{a}-C_{c}{ }^{a} b\right),
$$

where

$$
\begin{aligned}
C_{b c}^{a} & =\left(\partial_{\hat{b}} h_{\hat{a}}^{a}-\partial_{\hat{a}} h_{\hat{b}}^{a}\right) h_{b}{ }^{\hat{b}} h_{c}{ }^{\hat{a}} \\
& =h^{a}{ }_{\hat{a}}\left(\partial_{c} h_{b}{ }^{\hat{a}}-\partial_{b} h_{c}{ }^{\hat{a}}\right),
\end{aligned}
$$

where equation $\partial_{c}\left(h^{a}{ }_{\hat{a}} h_{a}{ }^{\hat{b}}\right)=\partial_{c}\left(\delta_{\hat{a}}^{\hat{b}}\right)=0$ and $\partial_{a}=h_{a}{ }^{\hat{b}} \partial_{\hat{b}}$ are used. Using the tetrad (9) and equaton (14), equaton (18) becomes

$$
\frac{d^{2} x^{a}}{d \tau^{2}}-\frac{e}{m} F^{a}{ }_{b} \frac{d x^{b}}{d \tau}=0,
$$

where $F_{a b}=\partial_{a} A_{b}-\partial_{b} A_{a}$ and $A_{a}$ is electromagnetic potential. Therefore, the Lorentz equation of motion is obtained using 4-dimensional Riemannian manifold $M$ defined by the metric $G_{\hat{a} \hat{b}}$.

\section{Covariance of the Lorentz equation of motion}

Motion equation (18) is form invariant with respect to local Lorentz transformations, under which [22], it is expressed as

$$
\frac{d^{2} x^{a^{\prime}}}{d \tau^{2}}+\omega^{a^{\prime}}{ }_{b^{\prime} c^{\prime}} \frac{d x^{b^{\prime}}}{d \tau} \frac{d x^{c^{\prime}}}{d \tau}=0,
$$

where $\omega^{a^{\prime}}{ }_{b^{\prime} c^{\prime}}$ transform as [21]

$$
\omega^{a^{\prime}}{ }_{b^{\prime} c^{\prime}}=\Lambda^{a^{\prime}}{ }_{a} \Lambda_{b^{\prime}}{ }^{b} \Lambda_{c^{\prime}}{ }^{c} \omega^{a}{ }_{b c}+\Lambda^{a^{\prime}}{ }_{d} \partial_{c^{\prime}} \Lambda_{b^{\prime}}{ }^{d},
$$

where $\Lambda^{a^{\prime}}{ }_{a}$ are local Lorentz transformations, and the relationship between $d x^{a^{\prime}}$ and $d x^{\hat{a}}$ is given by the $\Lambda^{a^{\prime}}{ }_{a}$,

$$
d x^{a^{\prime}}=\Lambda_{a}^{a^{\prime}} d x^{a} .
$$


Equation (22) is considered to describe the equation of motion in the different reference frame with coordinates $x^{a^{\prime}}$. Thus, because equation (18) is equivalent to the Lorentz equation of motion, the Lorentz equation of motion in the other reference frames can be studied using equation (22) and two cases are as follows.

For the case that the local Lorentz transformations are constant, equation (22) becomes

$$
\frac{d^{2} x^{a^{\prime}}}{d \tau^{2}}-\frac{e}{m} F^{a^{\prime}}{ }_{b^{\prime}} \frac{d x^{b^{\prime}}}{d \tau}=0
$$

where $F^{a^{\prime}}{ }_{b^{\prime}}=\Lambda^{a^{\prime}}{ }_{a} \Lambda_{b^{\prime}}{ }^{b} F^{a}{ }_{b}$, equation (25) is the Lorenz equation of motion in another inertial frame. When the local Lorentz transformations are time-dependent, the reference frame with coordinates $x^{a \prime}$ is a non-inertial frame. It is of significant importance that the motion equation in the non-inertial frame is studied using equation (22) depending on the corresponding local Lorentz transformations.

We consider a special non-inertial frame-the proper reference frame (comoving frame) that is attached to the charged particle in an electromagnetic field. Besides, the charged particle in the proper reference frame is always stationary. Thus, $d x^{a^{\prime}}$ is the differential coordinates of the proper reference frame, and $d x^{0^{\prime}}=d \tau$ and $d x^{i^{\prime}}=0$ in the proper reference frame. From the relation $\Lambda_{a^{\prime}}{ }^{a} d x^{a^{\prime}}=d x^{a}$, we have $\Lambda_{0^{\prime}}{ }^{a} d x^{0^{\prime}}=d x^{a}$, which becomes

$$
\Lambda_{0^{\prime}}{ }^{a}=\frac{d x^{a}}{d \tau} .
$$

The term $\Lambda^{a^{\prime}}{ }_{a} \partial_{c^{\prime}} \Lambda_{b^{\prime}} a \frac{d x^{b^{\prime}}}{d \tau} \frac{d x^{c^{\prime}}}{d \tau}$ in equation (22) is calculated,

$$
\begin{aligned}
\Lambda^{a^{\prime}}{ }_{a} \partial_{c^{\prime}} \Lambda_{b^{\prime}}{ }^{a} \frac{d x^{b^{\prime}}}{d \tau} \frac{d x^{c^{\prime}}}{d \tau} & =\Lambda^{a^{\prime}}{ }_{a} \partial_{0^{\prime}} \Lambda_{0^{\prime}}{ }^{a} \frac{d x^{0^{\prime}}}{d \tau} \frac{d x^{0^{\prime}}}{d \tau} \\
& =\Lambda^{a^{\prime}} a \frac{d^{2} x^{a}}{d \tau^{2}} .
\end{aligned}
$$

In the proper frame, equation (22) becomes

$$
\frac{d^{2} \chi^{a^{\prime}}}{d \tau^{2}}+\Lambda^{a^{\prime}}{ }_{a} \frac{d^{2} \chi^{a}}{d \tau^{2}}-\frac{e}{m} F_{b^{\prime}}^{a^{\prime}} \frac{d x^{b^{\prime}}}{d \tau}=0 .
$$

Using the Lorentz equation of motion, the latter two items in equation (28) satisfy equation

$$
L^{a^{\prime}}{ }_{a} \frac{d^{2} x^{a}}{d \tau^{2}}-\frac{e}{m} F_{b^{\prime}}^{a^{\prime}} \frac{d x^{b^{\prime}}}{d \tau}=0 .
$$

Thus, motion equation (22) becomes

$$
\frac{d^{2} \chi^{a^{\prime}}}{d \tau^{2}}=0
$$

We know that the proper reference frame (comoving frame) is the non-inertial reference frame, in which the charged particle is subjected to a fictitious force and a electromagnetic force. These two forces cancel each other out which result in the motion equation of the particle as $\frac{d^{2} x^{a^{\prime}}}{d \tau^{2}}=0$ in the proper reference frame. In our method, the motion equation of the particle is expressed as motion equation (22) in the proper reference frame. After being calculated, motion equation (22) becomes motion equation (28), in which the term $-\Lambda^{a^{\prime}}{ }_{a} d^{2} x^{a} / d \tau^{2}$ is fictitious acceleration and the term $\frac{e}{m} F^{a^{\prime}}{ }_{b^{\prime}} d x^{b^{\prime}} / d \tau$ is the electromagnetic acceleration, and these two forces cancel each other out. The result is reasonable in the proper reference frame.

\section{Conclusions}

To conclude, this work proposes the new metric (15) in flat spacetime to use for the curved worldline $x^{a}(\tau)$ of charged particles in an electromagnetic field. Then, we show that equation (18) is the motion equation of the charged particles and the equation is equivalent to the Lorentz equation of motion using the differential geometry of the metric (15). As the Lorentz equation of motion can describe the change of worldline $x^{a}(\tau)$, the motion equation (18) obtained shows that our model is self-consistent. Additionally, we have also investigated the local Lorentz covariance of the Lorentz equation of motion based on the equivalent equation (18), which is form invariant under the local Lorentz transformations. The important conclusion is that the Lorentz equation of motion in different inertial or non-inertial reference frames is related using equation (22). One example is that the motion equation (22) in the proper frame has been discussed and a rational prediction result has been obtained. In a sense, our method extends the Lorentz covariance of the Lorentz equation of motion to the local Lorentz covariance. Admittedly, local Lorentz covariance closely relates to general covariance in general relativity. Despite that general covariance has been proposed for numerous years, physicists do not have a unified understanding and the discussion continues for general covariance [23-28]. This research may provide a new point of view for understanding geometrizing electromagnetism and the relationship between electromagnetic interaction and general covariance.

The innovation of this paper is that the curve characteristic of the worldline of charged particles in an electromagnetic field is described by the metric (15), and the Lorentz equation of motion is achieved by using the Riemannian geometry defined by the metric. Our method has an interesting relationship with general relativity. The important similarity between general relativity and our 
method is that a 4-dimensional Riemannian manifold is used for studying interactions. Our method significantly differs from general relativity in the following ways:

(a) Our method investigates the relative motion of an object in flat spacetime. We introduce differential geometry to describe the curved worldline rather than curved spacetime. In the present method, the coordinates $\left\{x^{\hat{a}}\right\}$ represent the characteristics of a "curved" coordinate with respect to flat coordinates $\left\{x^{a}\right\}$, but the coordinates $\left\{x^{\hat{a}}\right\}$ are hypothetical.

(b) In general relativity, the metric does not depend on the properties of the object that is accelerated by a gravity indicating that objects of different masses in the gravitational effect have the same geodesic equation (equation of motion). However, in our method, the metric depends on the particle's charge-to-mass ratio $\mathrm{e} / \mathrm{m}$ properties. These results are significant, as charged particles with different $e / m$ ratios in an electromagnetic field have different worldlines, consequently introducing different metrics and different motion equations.

(c) In our method, the motion equation (18) is constructed based on the Ricci rotation coefficients rather than the Christoffel symbols. The Ricci rotation coefficients are employed to describe the physical laws in flat spacetime.

To some extent, the present research is significant to unify electromagnetism and gravitation with differential geometry.

Further studies of differential geometry are needed to perfect our model. At first, the study of the classical dynamics equation may be extended into quantum electrodynamics using differential geometry. Secondly, the problem that electromagnetic potential satisfies the Maxwell equation need to be studied in the framework of differential geometry. We believe that the methods and proofs described in this paper bring us a step closer to the final unified theory which will tightly associate differential geometry with fundamental electromagnetic interactions.

Acknowledgement: The work is supported by Open Foundation of Guizhou Provincial Key Laboratory of Radio Astronomy and Data Processing, Youth Innovation Promotion Association, Chinese Academy of Sciences under Grant No. 2016056, the Development Project of Science and Technology of Jilin Province under Grant No. 20180520077JH and the National Natural Science Foundation of China Nos. 11805022 and 11803057.

\section{References}

[1] Goenner H.F., On the history of unified field theories, Living Reviews in Relativity, 2004, 7, 1830-1923.

[2] Witten L., Geometry of gravitation and electromagnetism, Phys. Rev., 1959, 115, 206-214.

[3] Borchsenius K., Unified theory of gravitation, electromagnetism, and the yang-mills field, Phys. Rev. D, 1976, 13, 2707 2712.

[4] Ferraris M., Kijowski J., Unified geometric theory of electromagnetic and gravitational interactions, General Relat. Gravit., 1982, 14, 37-47.

[5] Vargas J. G., On the geometrization of electrodynamics, Found. Phys., 1991, 21, 379-401.

[6] Popławski N.J., Geometrization of electromagnetism in tetradspin gravity, Modern Phys. Let. A, 2009, 24, 431-442.

[7] Popławski N.J., Torsion as electromagnetism and spin, International J. Theor. Phys., 2010, 49, 1481-1488.

[8] Giglio J.F.T., Rodrigues Jr W.A., Gravitation and electromagnetism as geometrical objects of a riemann-cartan spacetime structure, Adv. Appl. Clifford Algebras, 2012, 22, 649-664.

[9] Shahverdiyev S.S., General geometry and geometry of electromagnetism, 2002, arXiv preprint hep-th/0205224.

[10] Olkhov O.A., New approach to geometrization of electromagnetic field, Amer. J. Phys. Appl., 2015, 3, 221-225.

[11] Duarte C.A., The classical geometrization of the electromagnetism, Int. J. Geom. Meth. Modern Phys., 2015, 12, 1560022.

[12] Synge J.L., Timelike helices in flat space-time, Proc. Royal Irish Acad., 1966, 65, 27-42.

[13] Honig E., Schucking E.L., Vishveshwara C.V., Motion of charged particles in homogeneous electromagnetic fields, J. Math. Phys., 1974, 15, 774-781.

[14] Caltenco J.H., Linares y M., Lopez-Bonilla J.L., Intrinsic geometry of curves and the lorentz equation, Czechoslovak J. Phys., 2002, 52, 839-842.

[15] Formiga J.B., Romero C., On the differential geometry of timelike curves in minkowski spacetime, Amer. J. Phys., 2006, 74, 1012-1016.

[16] Misner C.W., Thorne K.S., Wheeler, J.A., 1973, San Francisco, W.H. Freeman and Co.

[17] Ni W.T., Zimmermann M., Inertial and gravitational effects in the proper reference frame of an accelerated, rotating observer, Phys. Rev. D, 1978, 17, 1473-1476.

[18] Nelson R.A., Generalized lorentz transformation for an accel- erated, rotating frame of reference, J. Math. Phys., 1987, 28, 2379 2383.

[19] Aldrovandi R., Barros P., Pereira J., The equivalence principle revisited, 2003, Found. Phys., 33, 545-575.

[20] Mei T., A new variable in general relativity and its applications for classic and quantum gravity, 2006, arXiv preprint grqc/ 0611063.

[21] De Felice F., Clarke C.J., Relativity on curved manifolds, Cambridge University Press, 1992.

[22] Carroll S.M., Lecture notes on general relativity, 1997, arXiv preprint gr-qc/9712019.

[23] Ellis G.F.R., Matravers D.R., General covariance in general relativity? General Relat. Gravit., 1995, 27, 777-788.

[24] Zalaletdinov R., Tavakol R., Ellis G.F.R., On general and restricted covariance in general relativity, General Relat. Gravit., 
1996, 28, 1251-1267.

[25] Gron 0., Voyenli K., On the foundation of the principle of relativity, Foundations of Physics, 1999, 29, 1695-1733.

[26] Brown H.R., Physical relativity: Space-time structure from a dynamical perspective, 2005, Oxford University Press on Demand.

[27] Westman H., Sonego S., Events and observables in generally invariant spacetime theories, Found. Phys., 2008, 38, 908-915.

[28] Chamorro A., On the meaning of the principle of general covariance, Int. J. Theor. Phys., 2013, 52, 117-129.

[29] Pauli W., Theory of relativity, 1958, Courier Dover Publications.

\section{Appendix A}

$\sqrt{1-v^{2}}$ is associated with the electromagnetic potential of a charged particle accelerated by an electromagnetic field. According to relativistic mechanics [29],

$$
\frac{d U^{i}}{d \tau}=k^{i}=\frac{e}{m \sqrt{1-v^{2}}}(\vec{E}+\vec{v} \times \vec{B}),
$$

and

$$
\frac{d U^{0}}{d \tau}=k^{i} v^{i}
$$

where $U^{0}=1 / \sqrt{1-v^{2}}$ and $U^{i}=v^{i} / \sqrt{1-v^{2}}$. Combining equation (A.1) and (A.2), it can see that $U^{0}$ satisfies the relationship

$$
d U^{0}=\frac{e}{m}\left(-\frac{\partial A_{0}}{\partial x^{i}}+\frac{\partial A_{i}}{\partial x^{0}}\right) d x^{i} .
$$

$U^{0}$ can be divided into the contributions of scalar field $A_{0}$ and vector field $A_{i}$, that is $U^{0}=U^{0}{ }_{e}+U^{0}{ }_{m}, d U^{0}{ }_{e}=$ $-\frac{e}{m} \frac{\partial A_{0}}{\partial x^{i}} d x^{i}$ and $d U^{0}{ }_{m}=\frac{e}{m} \frac{\partial A_{i}}{\partial x^{0}} d x^{i}$. For the interaction of a charged particle with a scalar field $A_{0}$, the following relationship is obtained,

$$
U^{0}{ }_{e}=C-\frac{e}{m} A_{0},
$$

where $C$ is a constant determined by the initial conditions. However, $U^{0}{ }_{m}$ is not easy to obtain. It is considered that the effect of vector field $A_{i}$ in equation (A.3) can always be replaced by a function of time at some fixed spacetime points. Therefore, $U^{0}{ }_{m}$ is rewritten as a function of time. We set

$$
U^{0}=\frac{1}{\sqrt{1-v^{2}}}=1-\frac{e}{m} A_{0}+Q(t),
$$

where $Q(t)$ is the time function and determined by the vector field $A_{i}$. Based on the above argument, $\sqrt{1-v^{2}}$ becomes a function of the electric potential $A_{0}$ and time $t$. 